\title{
Kinematic and kinetic analysis of planned and unplanned gait termination in children
}

\author{
Sarah T. Ridge \\ Brigham Young University - Provo, sarah_ridge@byu.edu \\ John Henley \\ Kurt Manal \\ University of Delaware \\ Freeman Miller \\ James G. Richards
}

Follow this and additional works at: https://scholarsarchive.byu.edu/facpub

Part of the Exercise Science Commons

\section{Original Publication Citation}

Ridge, S, Henley, J, Manal, K, Miller, F \& Richards, J. (2013) Kinematic and kinetic analysis of planned and unplanned gait termination in children. Gait and Posture, 37(2), 178-182.

\section{BYU ScholarsArchive Citation}

Ridge, Sarah T.; Henley, John; Manal, Kurt; Miller, Freeman; and Richards, James G., "Kinematic and kinetic analysis of planned and unplanned gait termination in children" (2013). Faculty Publications. 2016. https://scholarsarchive. byu.edu/facpub/2016 accepted for inclusion in Faculty Publications by an authorized administrator of BYU ScholarsArchive. For more information, please contact ellen_amatangelo@byu.edu. 


\title{
Kinematic and kinetic analysis of planned and unplanned gait termination in children
}

Sarah Trager Ridge, John Henley, Kurt Manal, Freeman Miller, James G.Richards

\begin{abstract}
Gait termination is a task which requires people to alter momentum and stabilize the body. To date, many of the kinematic and kinetic characteristics of gait termination have not been reported, making it difficult for clinicians to design interventions to improve the ability to terminate gait quickly and efficiently. Therefore, the purpose of this study was to describe the lower body mechanics of healthy children as they performed walking trials, planned stopping trials, and unplanned stopping trials. Kinematic and kinetic data were collected from 15 healthy children between the ages of 11 and 17 years (14.3 \pm 2.1 years). The timing and magnitude of peak sagittal plane joint angles and moments were compared across the three conditions for the leg that led the stop step. Most differences were found when comparing unplanned stopping to both walking and planned stopping. During unplanned stopping, most subjects used either a hip/knee extension strategy or hip/knee flexion strategy to stabilize and perform the stopping task. The magnitudes of the peak hip extension moment and peak knee flexion angle were significantly greater, while the peak plantarflexion moment was significantly smaller during unplanned stopping than walking and planned stopping. The peak plantarflexion moment occurred significantly earlier during the stop stance phase of planned and unplanned stopping than during walking. This suggests that the ability to create sufficient joint moments in a short period of time is essential to be able to stop quickly and safely. Therefore, possible treatments/interventions should focus on ensuring that patients have appropriate strength, power, and range of motion.
\end{abstract}

\section{Keywords}

Gait, Gait termination, Children

\section{Introduction}

The mechanics of healthy gait have been extensively studied. Researchers are now focusing on other aspects of human gait, such as gait initiation [1]. However, the mechanics of gait termination have not been thoroughly examined. Gait termination requires the ability to alter momentum and stabilize the body quickly. In addition, the ability to terminate gait quickly and 
efficiently has implications for safety when moving in environments that may require quick reactions. The ability to stop before walking into an object, or into the path of an oncoming object, is crucial to an individual's safety.

Previous research on gait termination has focused primarily on muscle activation and ground reaction forces; few studies have examined joint kinetics. Lynch and Robertson [2] found similarities in lower limb joint moments between planned stopping and walking. They stated that the ankle plantarflexors, knee extensors, and hip flexors of the lead leg are instrumental in slowing forward motion and successfully terminating gait. They did not examine unplanned stops. Hase and Stein [3] identified three mechanisms used to terminate gait during unplanned stopping tasks when participants were not aware of when or where they would be asked to stop. The first mechanism was to increase the braking forces under the limb that leads the stopping step. Second, propulsive forces under the trailing limb were decreased compared to a typical walking step. Third, if the first two mechanisms were not strong enough or the decision to stop was made too late into the stop cycle, people rose onto their toes in an effort to convert kinetic energy to potential energy [3]. In another study that reported data from an unplanned stop, transfemoral amputees were found to terminate gait with a more extended hip and knee in their lead leg than their able bodied counterparts [4]. This may help compensate for the amputees' inability to produce the larger anterior-posterior forces that are required for braking.

What is still not well understood, however, are the kinetic strategies used at individual joints that produce these mechanisms. In fact, we are unaware of any studies to date that report joint moment data during unplanned stopping tasks. Understanding the joint kinetics required to complete planned and unplanned stopping tasks is important in order to ensure that surgical and other therapeutic interventions designed to improve walking also take into account the joint kinetics needed to safely terminate gait.

The purpose of this study was to describe the lower body mechanics of healthy children while performing unplanned and planned stopping tasks, both necessary activities of daily living. A range of ages of children who were old enough to have matured gait were chosen for this study in order to maintain relevance of our data to a typical population which may have difficulty with stopping - children with cerebral palsy (often post surgery) [5]. Pilot data collected on seven healthy children showed conflicting kinematic strategies during stopping, specifically regarding hip and knee flexion angles in the lead limb. Therefore, the hypotheses for this study were stated in null format. Specifically, the hypotheses stated that lower body kinematics and kinetics during unplanned and planned stopping would be no different than those exhibited during steady-state walking.

\section{Methods}

\subsection{Data collection}

15 healthy children between the ages of 11 and 17 years (average age $14.3 \pm 2.1$ years) participated. Children and their parents signed informed assent/consent forms approved by the institution's Human Subjects Review Board. 
Height, weight, and arm dominance were recorded at the beginning of each subject's visit. Reflective markers were applied to anatomical landmarks to create a 12 segment full body (no head) model according to a modified Helen Hayes marker set. Video data was collected at $60 \mathrm{~Hz}$ using 10 Eagle Digital Motion Analysis Cameras (Motion Analysis, Santa Rosa, CA). Force plate data was collected through the same software that collected the video data (Cortex 1.1) from four AMTI force plates (Advanced Mechanical Technology, Inc., Watertown, MA) at a rate of $960 \mathrm{~Hz}$.

Subjects walked across the room an average of 30 times, including 3-5 trials to establish the subject's preferred walking speed. These trials were also used as the "walk-through" trials for comparison with the kinematics and kinetics of the stopping trials. After the preferred speed was determined, subjects walked across the room at their preferred speed while looking at a screen positioned at the end of the walkway. Subjects monitored their velocity by watching an image that included two sliders on an analog scale: one slider represented their actual position, while the other slider represented their target position. The target position was where they should be if they were walking at the speed established during the initial walking trials [6]. The volume provided subjects at least three steps to stabilize their walking speed before reaching the force plates.

Subjects performed stopping trials under two different conditions: unplanned stopping and planned stopping, both at their preferred speed. During the planned stopping trials, subjects were told to stop on a specific force plate, bring their feet together, and freeze. For the unplanned stopping trials, subjects were not told beforehand which force plate to stop on. Instead, they were given a signal to stop during the trial. The velocity sliders were replaced by a large STOP sign and the screen turned red. Subjects were instructed to "freeze" as soon as they saw the stop sign, then hold that position until they were told to move again. The stop sign appeared at foot contact of the dominant leg. At least three trials were collected when the leading foot landed on one of the four randomly selected force plates. Foot contacts were determined in real time by streaming the marker data from the motion analysis software to custom LabView software. The LabView software read the coordinates frame-by-frame for the sacral marker and the heel marker on the dominant foot. These two markers were used to calculate foot contact, and different foot contacts (first, second, or third) were used to trigger the stop signal in each trial [7].

\subsection{Data analysis}

Marker data were tracked and filtered with a $6 \mathrm{~Hz}$ Butterworth filter. Variables including lower body joint angles, moments and total body (minus the head) center of mass position were calculated using Visual 3d (C-Motion, Inc., Germantown, MD). All moments were normalized to each subject's height and body mass. Total body center of mass was calculated in Visual 3d using inertial properties described by Hanavan [8].

Subjects were considered stopped (COM stop) when the forward velocity of their center of mass dropped below $5 \%$ of their preferred forward velocity. Time to stop was calculated as the time that elapsed between the stop signal and the COM stop.

Only trials in which subjects were able to stop within one step of receiving the signal were analyzed. When the subjects were able to stop within one step, the legs were referred to as the trailing limb and the lead limb (in reference to the final positions of the legs after stopping) for 
further analysis (see Fig. 1.1). Lead limb peak hip flexion, knee flexion, and plantarflexion angles were reported during the stop step (terminal step). For every stop trial where the stop step occurred on a force plate, the peak plantarflexion moment, peak knee extension moment, and peak hip extension moment were reported. Peaks from stop steps were chosen during the terminal stance phase - between terminal foot contact and COM stop. Peaks were averaged from up to three individual trials prior to statistical analysis. The same peak moments were calculated for the planned and unplanned stopping trials.

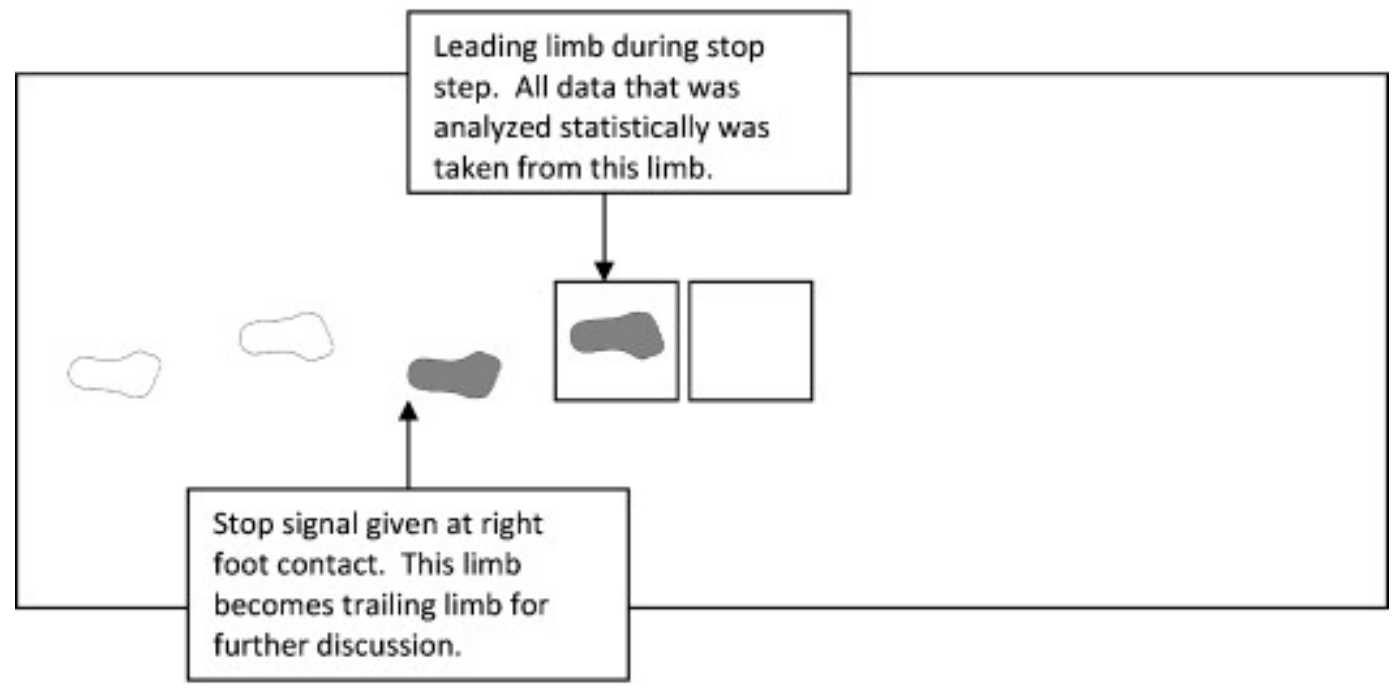

Fig. 1.1. Overhead view of data collection volume as a right arm dominant subject completed an unplanned stopping trial. The dark gray footsteps represent a subject's feet in the stopped position. The squares in the middle of the volume are force plates. If the subject had been left arm dominant, the signal would have been given at left foot contact and the right foot would have been the lead leg on the force plate during the stop step.

The timing of the peaks was also recorded. For walk-through trials, the timing of the peak was calculated as a percentage of the stance phase. For the stopping trials, the timing of the peak was calculated as a percentage of the final "stance" phase (stop stance) - from terminal foot contact (the last heel strike of the lead leg) until the COM stopped forward motion.

Statistical analyses included repeated measures ANOVAs across all conditions with an alpha level of $p<.01$. If statistical differences were found, a Tukey's post hoc test was run to determine where the differences occurred.

\section{Results}

Subjects were able to stop within one step after receiving the stop signal in $93.8( \pm 9.33) \%$ of trials. Individual subjects ranged from a $68.75 \%$ to $100 \%$ success rate of stopping in one step during these trials. Subjects required an average of $1.06( \pm .07) \mathrm{s}$ to come to a complete stop after presentation of the stop signal. 
Some differences were found in joint angle and moment data when comparing unplanned stopping to walking and planned stopping (Table 1.1). Specifically, peak knee flexion angle and peak hip extension moment of the leading leg were significantly greater during the unplanned stop step than during planned stop steps and walking steps. Peak hip flexion angle was significantly greater during the unplanned stop step than during the planned stop step, but no different than during walking steps. The peak plantarflexion moment was significantly different across all conditions.

Table 1.1. Leading leg (non-dominant) joint angle and moment data from the stop step during walking steps from walk-through trials, unplanned and planned stopping trials from walk-through trials. $n=$ the number of subjects who completed at least two stopping trials.

$\begin{array}{lll}\text { Walking step } & \begin{array}{l}\text { Unplanned stop step } \\ (n=15)\end{array} & \begin{array}{l}\text { Planned stop step } \\ (n=15)\end{array}\end{array}$

\begin{tabular}{|c|c|c|c|c|}
\hline $\begin{array}{l}\text { Peak knee flexion angle } \\
\text { (degrees) }\end{array}$ & & $24.5 \pm 7.78^{\mathrm{b}}$ & $33.9 \pm 10.1^{\frac{\mathrm{a}, \mathrm{c}}{\mathrm{c}}}$ & $22.3 \pm 8.48^{\mathrm{b}}$ \\
\hline Peak hip flexion angle ( & degrees) & $26.4 \pm 4.39$ & $29.9 \pm 6.56^{\mathrm{c}}$ & $23.1 \pm 5.49 \underline{b}$ \\
\hline $\begin{array}{l}\text { Peak ankle plantarflexio } \\
\text { moment }(\mathrm{Nm} / \mathrm{kg} \mathrm{m})\end{array}$ & & $.803 \pm .133^{\mathrm{b}, \mathrm{c}}$ & $.599 \pm .100^{\mathrm{a}, \mathrm{c}}$ & $.448 \pm .132^{\frac{\mathrm{a}, \mathrm{b}}{\mathrm{b}}}$ \\
\hline $\begin{array}{l}\text { Peak knee extension mo } \\
(\mathrm{Nm} / \mathrm{kg} \mathrm{m})\end{array}$ & ment & $.298 \pm .158$ & $.211 \pm .263$ & $.185 \pm .163$ \\
\hline $\begin{array}{l}\text { Peak hip extension mom } \\
\text { (Nm/kg m) }\end{array}$ & & $.293 \pm .057 \underline{\mathrm{b}}$ & $.432 \pm .105^{\mathrm{a}, \mathrm{c}}$ & $.252 \pm .075^{\underline{b}}$ \\
\hline Timing & $\begin{array}{l}\text { \% Stan } \\
\text { step }(n\end{array}$ & $\begin{array}{l}\text { e- walking } \\
\text { 15) }\end{array}$ & $\begin{array}{l}\text { \% Stance - unplanned } \\
\text { stop step }(n=15)\end{array}$ & $\begin{array}{l}\% \text { Stance - planned } \\
\text { stop step }(n=15)\end{array}$ \\
\hline $\begin{array}{l}\text { Peak knee flexion } \\
\text { angle }\end{array}$ & $27.9 \pm 5$ & & $29.9 \pm 8.41$ & $22.6 \pm 7.49$ \\
\hline Peak hip flexion angle & $8.12 \pm 4$ & $46^{\underline{b}}$ & $24.9 \pm 15.9^{9^{\mathrm{a}, \mathrm{c}}}$ & $7.83 \pm 4.15^{\underline{b}}$ \\
\hline $\begin{array}{l}\text { Peak ankle } \\
\text { plantarflexion moment }\end{array}$ & $83.0 \pm 1$ & $52^{\mathrm{b}, \mathrm{c}}$ & $33.3 \pm 13.4^{\mathrm{a}, \mathrm{c}}$ & $42.1 \pm 7.21^{\mathrm{a}, \mathrm{b}}$ \\
\hline $\begin{array}{l}\text { Peak knee extension } \\
\text { moment }\end{array}$ & $27.2 \pm 2$ & & $25.6 \pm 8.72$ & $19.9 \pm 4.72^{\underline{a}}$ \\
\hline
\end{tabular}




$\begin{array}{llll}\text { Timing } & \begin{array}{l}\text { \% Stance }- \text { walking } \\ \text { step }(\boldsymbol{n}=\mathbf{1 5})\end{array} & \begin{array}{l}\text { \% Stance }- \text { unplanned } \\ \text { stop step }(\boldsymbol{n}=\mathbf{1 5})\end{array} & \begin{array}{l}\text { \% Stance }- \text { planned } \\ \text { stop step }(\boldsymbol{n}=15)\end{array} \\ \begin{array}{l}\text { Peak hip extension } \\ \text { moment }\end{array} & 8.91 \pm 4.31^{\mathrm{b}} & 11.4 \pm 14.7^{\mathrm{a}, \mathrm{c}} & 6.21 \pm 4.41^{\mathrm{b}}\end{array}$

A Differenfrom walking. B Different from unplanned. C Different from planned.

The timing of these peaks was significantly different between conditions. Peak hip flexion angle occurred significantly later during the "stance" phase of unplanned stopping than during planned stopping or walking steps. There was substantial variability in this measure across subjects as some subjects achieved peak hip flexion angle very early in the trial, while others continued flexion until COM stop. The peak plantarflexion moment occurred significantly earlier during planned and unplanned stop steps than during walking steps.

Peak knee extension moments also occurred significantly earlier in the planned stopping condition than during walking, but not during the unplanned stopping condition (although trending toward significance at $p=.012$ ).

\section{Discussion}

Throughout this study, only sagittal plane angles and moments were analyzed. Previous research has shown that the most important factor for stopping is the ability to produce sufficient anterior-posterior ground reaction forces [3,9-12]. Therefore, it seems that examining the kinematics and kinetics of the lower body that contribute to this aspect of stopping should provide the most relevant information about how the body is able to produce a stop.

Our results indicated that planned stopping was similar to walking for the majority of subjects. The exception to this occurred at the end of the stance phase. Subjects executing planned stops tended to demonstrate earlier and increased hip and knee flexion of the lead leg before bringing their legs together to stabilize in a position similar to an upright, static standing posture.

Unplanned stopping demonstrated more profound differences during the stop stance phase when compared to the walking stance phase or the planned stopping stance phase. At the ankle, most subjects (13 of 15) made foot contact in a more plantarflexed position for the unplanned stop than during walking (see Fig. 1.2a). 

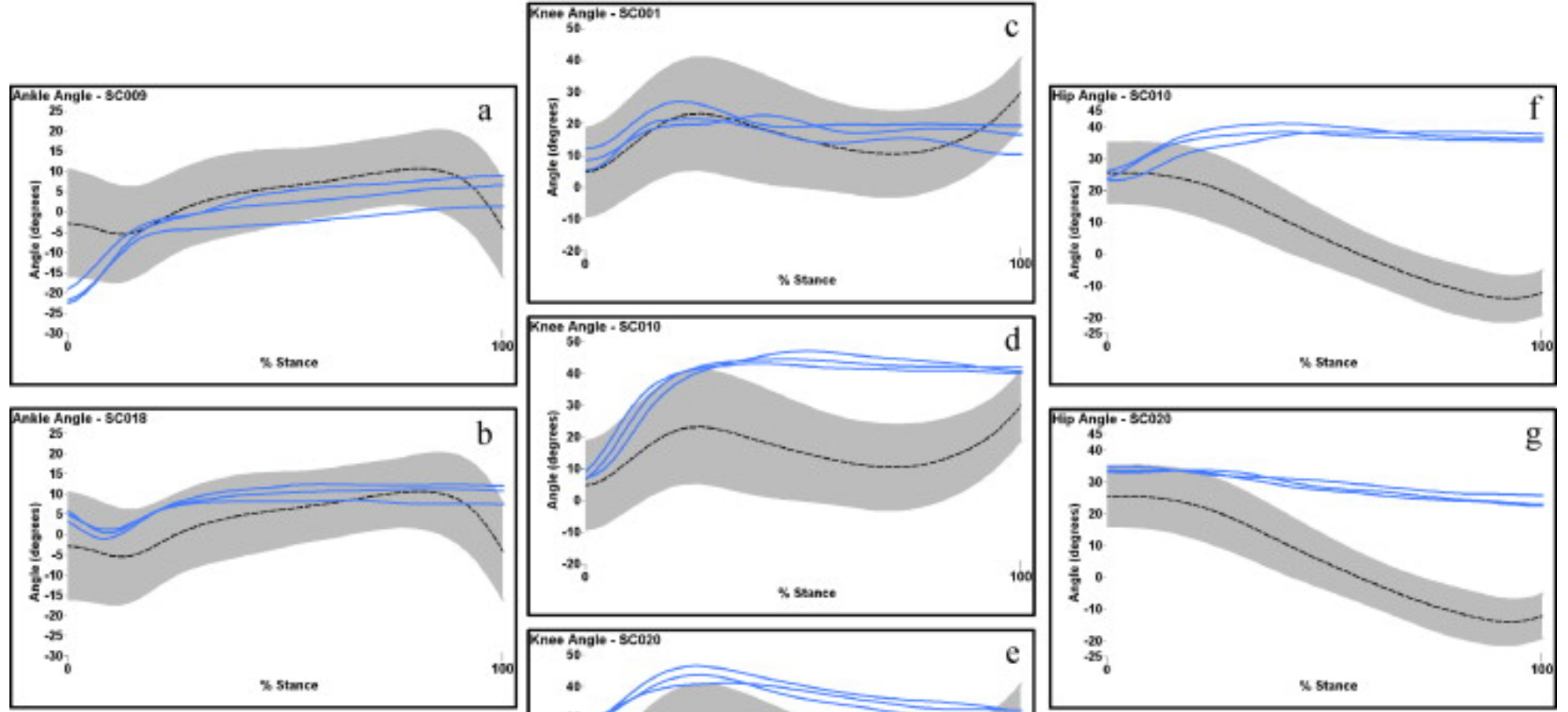

Fig. 1.2. Joint angles from representative subjects. The blue, solid lines on each graph show a representation of a strategy used by some subjects during an unplanned stopping trial. These data are from a representative individual's trials. The black, dashed line is the average of all subjects' walking trials. The gray shaded area represents the group average for walking $\pm 2 \mathrm{SD}$. The $x$-axis represents the walking stance phase or stop stance phase normalized to 50 points. (For interpretation of the references to color in this figure legend, the reader is referred to the web version of the article.)

The plantarflexion moment at foot contact during unplanned stopping was greater during the stop step than during walking in most subjects. These moments increased until about $15 \%$ of the stop stance phase, after which they stabilized until the COM stopped moving forward (see Fig. 1.3 a and $b$ ). The peak plantarflexion moments during both planned and unplanned stopping were significantly smaller than those produced during walking, as was the amount of time the person had to create those moments. Gastrocnemius weakness, present in some pathologies, can cause difficulty performing quick plantarflexion and subsequently controlling the plantarflexion moment, especially during braking. Therefore, this may be a consideration for physical therapists and surgeons as they are planning treatment. 

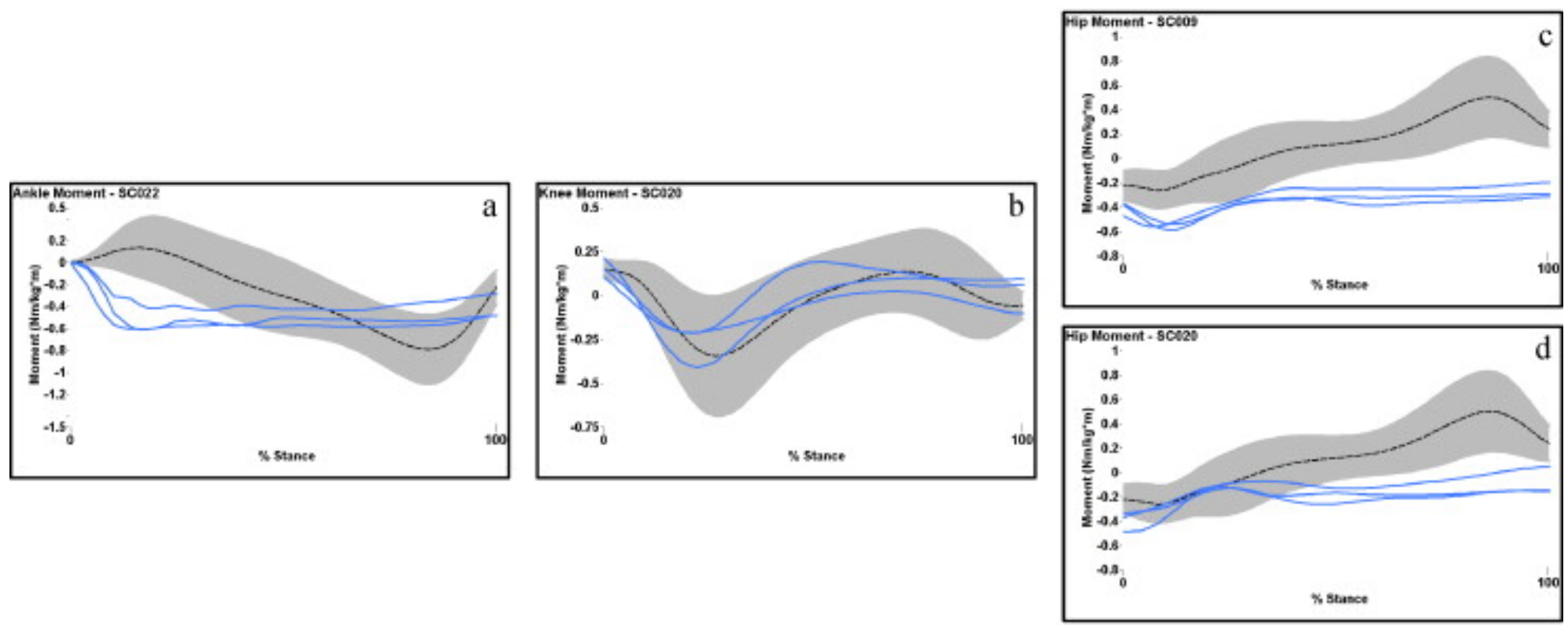

Fig. 1.3. Joint moments from representative subjects. The blue, solid lines on each graph show a representation of a strategy used by some subjects during an unplanned stopping trial. These data are from a representative individual's trials. The black, dashed line is the average of all subjects' walking trials. The gray shaded area represents the group average for walking $\pm 2 \mathrm{SD}$. The $x$-axis represents the walking stance phase or stop stance phase normalized to 50 points. (For interpretation of the references to color in this figure legend, the reader is referred to the web version of the article.)

During the planned stopping trials, subjects were instructed to stop with their feet together on the force plate. The different stopping positions during planned and unplanned stopping represent a limitation of this study. For approximately one-third of the trials, the peak plantarflexion moment occurred just before contralateral foot contact on the same force plate. In these cases, this may or may not have been the true peak, as the plantarflexion moment continued to increase after contralateral foot strike. However, this increase was likely due, at least in part, to the contact made by the other foot and the shift of location of the ground reaction force vector toward the location of the trailing foot. Therefore, the peak ankle plantarflexion moment was either the true peak (if the moment decreased again before contralateral foot contact) or at the frame just prior to contralateral foot contact.

Subjects demonstrated a variety of knee strategies during unplanned stopping (see Fig. 1.2c-e). Subjects who landed and stabilized with greater knee flexion tended to reach COM stop more quickly. All subjects continued knee flexion through the early part of the stop stance phase. Some subjects ( 3 of 15) stayed in deep knee flexion to stabilize (see Fig. 1.2d), while others (9 of 15) began to extend again prior to COM stop (see Fig. 1.2c and e). The remaining three subjects did not show clear preferences for any specific strategy.

Knee joint moments during unplanned stopping generally followed the same pattern as during walking. The magnitude of the knee extension moments was not significantly different across the 
three conditions (see Fig. 1.3c). It appears that if children are able to walk, knee extensor weakness should not factor into their ability to terminate gait. The peak knee extension moment was earlier during planned stopping than walking, however. Therefore, during treatment clinicians should consider the need for earlier muscle activation.

Two hip strategies prevailed during unplanned stopping, though there was no significant difference in time to stop between the two strategies. One strategy, used by six subjects, was to land, flex the hip, then stabilize in a position close to the peak hip flexion angle (see Fig. 1.2f). The other strategy, used by eight subjects, was to land, quickly flex, then return to a more extended position (see Fig. 1.2g). Not all subjects increased hip flexion after making contact; however, by the time these subjects stabilized, they all exhibited greater hip extension than at foot contact. One subject did not show a clear preference for either strategy throughout the stopping trials. These results suggest that therapists may develop training programs for stopping based on either strategy, depending on which may work best for each patient.

Hip joint moments during planned stopping were similar to those recorded during walking trials. However, during unplanned stopping, subjects had a significantly larger peak hip extension moment than during planned stopping or walking. This occurred at approximately the same time during the stance phase in all conditions. About half of the subjects maintained a larger hip extension moment after the peak when compared to walking (see Fig. 1.3c). Others showed hip extension moment patterns similar to walking until about $30 \%$ of the stop stance phase (see Fig. 1.3d). As previously stated, some subjects continued to increase hip flexion throughout the stop stance phase, though most slightly decreased hip flexion after reaching an early peak. Therefore, the strength of the child's hip extensors and the ability to stabilize and maintain the hip extension moment may be necessary for successful gait termination.

\section{Summary}

With the exception of the peak plantarflexion moment and the timing of the peak knee extension moment, the magnitude and timing of the lower body joint angles and moments analyzed in this study were not significantly different between planned stopping and walking. More differences were seen in joint kinematics and kinetics when comparing unplanned stopping to walking and planned stopping. Therefore, our null hypotheses were rejected.

It appears that there are two hip and knee strategies employed by the majority of subjects during unplanned stopping. Most of the group of subjects that stabilized by extending the hip also extended the knee while stabilizing after reaching peak knee flexion. Meanwhile, the group of subjects that stabilized by locking the hip into flexion also locked the knee into flexion while stabilizing and stopping the forward motion of the center of mass. Whichever strategy was used, differences between walking steps and the stop step occurred very early during the stance phase.

Understanding the relationship between how the joints work together to produce an unplanned stop is important to determining why some children have difficulty with this task. Additionally, with an understanding of the strength and/or range of motion necessary to terminate gait, clinicians can be more aware of how to assist children with performing this task. Using this research as a basis for understanding the mechanics of stopping in typically developing children, 
future research should focus on which strategies are used by pathological populations who may have difficulty with stopping tasks, such as individuals with cerebral palsy.

\section{Conflict of interest statement}

There are no conflicts of interest or acknowledgements to report.

\section{References}

[1] Bishop M, Brunt D, Pathare N, Patel B. The effect of velocity on the strategies used during gait termination. Gait \& Posture 2004;20(2):134-9.

[2] Lynch J, Robertson D. Biomechanics of Planned Gait Termination. In: Proceedings of the XXI Congress of International Society of Biomechanics; 2007.

[3] Hase K, Stein RB. Analysis of rapid stopping during human walking. Journal of Neurophysiology 1998;80(1):255-61.

[4] Vrieling AH, van Keeken HG, Schoppen T, Otten E, Halbertsma JPK, Hof AL, et al. Gait termination in lower limb amputees. Gait \& Posture 2008;27(1):82-90.

[5] Ganley KJ, Powers CM. Gait kinematics and kinetics of 7-year-old children: a comparison to adults using age-specific anthropometric data. Gait \& Posture 2005;21(2):141-5.

[6] Ridge S, Richards JG. Real-time feedback as a method of monitoring walking velocity during gait analysis. Gait \& Posture 2011.

[7] Zeni JA, Richards JG, Higginson JS. Two simple methods for determining gait events during treadmill and overground walking using kinematic data. Gait \& Posture 2008;27(4):710-4.

[8] Hanavan Jr EP. A mathematical model of the human body. Amrl-Tr-64-102. Army Medical Research Lab Technical Report 1964;1-149.

[9] Meier MR, Desrosiers J, Bourassa P, Blaszczyk J. Effect of Type II diabetic peripheral neuropathy on gait termination in the elderly. Diabetologia 2001;44(5):585-92.

[10] Jaeger RJ, Vanitchatchavan P. Ground reaction forces during termination of human gait. Journal of Biomechanics 1992;25(10):1233-6.

[11] Bishop M, Brunt D, Marjama-Lyons J. Do people with Parkinson’s disease change strategy during unplanned gait termination? Neuroscience Letters 2006;397(3):240-4.

[12] Perry SD, Santos LC, Patla AE. Contribution of vision and cutaneous sensation to the control of centre of mass (COM) during gait termination. Brain Research 2001;913(1):27-34. 